\title{
Survey on a Trust-Aware System for Personalized User Recommendations in Social Networks
}

\author{
Supriya $S^{1}$, Saravanan $D^{2}$ \\ Information Technology, IFET College of Engineering, Villupuram, India ${ }^{1}$ \\ Assistant Professor, Information Technology, IFET College of engineering, Villupuram, India ${ }^{2}$
}

\begin{abstract}
Trust is an important part in a social network from security point of view. Online video sharing systems is the most popular and provide features that allow users to post a video in a web page. These features provide opportunities for a user to introduce polluted content into the system. Spammersmay post an unrelated video aiming at increasing the likelihood of the responsebeing viewed by a larger number of users. Multimedia recommendation system recommends video based on the user behavior which reduces network overhead and speed up the recommendation process. The proposed approach can recommend desired services with high precision, high recall and low response delay. To avoid the explosion of networkoverhead, user-behavior-based clustering is performed. If unrelated content is displayed in our web page, then we spam that video content. If more users spam all video content from same provider, then the provider will be deleted from server.
\end{abstract}

Keywords: social networks, recommendation, personalization, trust.

\section{I.INTRODUCTION}

With the recent emerging of social networks internet users based summary or transactionbased association analysis. became directly interconnected to share all kind of However, in many interesting applications, one may want information. Social web sites capture and display content to analyze the changes of measures in multidimensional generated by untrusted users, which can range from photos space.

to text to urls. Social web sites have four important characteristics relevant to our discussion of spam: one controlling entity, well-defined interactions, identity,multiple interfaces. These characteristics substantially change the dynamic of the adversarial relationship between service providers and spammers. The social semantic web can be seen as a web of collective knowledge systems. It provides useful information based on people's contributions. The approach of social semantic web is pragmatic because it uses humanly created (loose) semantics, such as semiformal ontologies or collaborative tagging. Thus, social semantic web effectively complements the fully automated semantics and formal ontologies of semantic web. Social recommender systems allow for the interoperability of system resources and the homogeneity of the representation of information, facilitate necessary means to generate descriptions enriched by web services, and permit their discovery by software agents. Therefore, semantic recommender systems can comprise very useful mechanisms in the field of semantic web, to facilitate information discovery. Discovery enables users to find new content of their interest that is shared by other users. Nowadays, collaborative tagging services proliferate on the web. The social semantic web can be seen as a web of collective knowledge systems. It provides useful information based on people's contributions. Recently, there have been growing interests in multidimensional analysis of relational databases, transactional databases, and data warehouses. Most of such analysis involve data cube-

\section{LITERATURE SURVEY}

In this section we are focus on different techniques used in recommendation and spammer detection system. [1] Fighting Spam on Social Websites: Identification-based methods proceed in two phases.In the first, likely spam is identified. Users can either manually identify spam, or the system owner can use pattern-based classification. In the second phase, the system interfaces take into account the likely spam and either delete it and then compute their results, or display particular results as likely spam. For these methods, we can treat the corpus as a set of objects with associated attributes. In email spam, the messages are objects and the headers areattributes. In Web spam, the Web pages are objects, and attributes might be inlinks, outlinks, page content, and various external metadata (such as the speed of a page rank increase or how many domain names resolve to the same IP). Social Web sites will often focus on a few types of objects, such as photos or urls, and the attributes will then pertain to these objects or their relationships. [2] Item Recommendation in Collaborative Tagging Systems: A data model is proposed from collaborative tagging systems with three-mode tensors, in order to capture the three-way correlations between users, tags, and items. By applying multiway analysis, latent correlations are revealed, which help to improve the quality of recommendations. Moreover, a hybrid scheme is proposed that additionally considers content-based information that is extracted from items. Experimental comparison, using data from a real 
collaborative tagging system (Last.fm), against both recent tag-aware and traditional (non tag aware) item recommendation algorithms indicates significant improvements in recommendation quality. [3] Mining Constrained Gradients in Large Databases: liveset-Driven algorithm finds all good gradient-probe cell pairs in one search pass. It utilizes measure-value analysis and dimension-match analysis in a set-oriented manner, to achieve bidirectional pruning between the sets of hopeful probe cells and of hopeful gradient cells. Moreover, it adopts a hypertree structure and an H-cubing method to compress data and to maximize sharing of computation. [4] Computing And Applying Trust In Web-Based Social Networks: For each rating scheme, the algorithms are built upon the defined notions of trust.

Each is then analyzed theoretically and with respect to simulated and actual trust networks to determine how accurately they calculate the opinions of people in the system. These calculations are then put to use in two applications. Filmtrust is a website that combines trust, social networks, and movie ratings and reviews. Trust is used to personalize the website for each user, displaying recommended movie ratings, and ordering reviews by relevance. [5] Predicting Positive and Negative Links in Online Social Networks: the signs of links in the underlying social networks can be predicted with high accuracy, using models that generalize across this diverse range of sites.

These models provide insight into some of the fundamental principles that drive the formation of signed links in networks, shedding light on theories of balance and status from social psychology; they also suggest social computing applications by which the attitude of one user toward another can be estimated from evidence provided by their relationships with other members of the surrounding social network.

\section{III.CONCLUSION}

Online trading platform possessing high flexibility, high reliability, low-level transparency, security features. Based on the user interests and report of the media content in the system, a collaborative filtering recommendation is used for video recommendation.A computing platform distributed in large-scale data center. The proposed system recommends channels based on their viewing pattern and interests. The users just need to select the channel that they want to watch.Promoters and spammers can pollute video retrieval feature of online video SNs, compromising not only user satisfaction with the system, but also the usage of system resources and the effectiveness of content delivery mechanisms such as caching and content delivery networks. We here proposed an effective solution that can help system administrators to detect spammers and promoters in online video SNs.

\section{IV.FUTURE WORK}

In future research efforts, we will focus on those characteristics of upcoming intelligent devices such as iPhone, iPad etc.

\section{REFERENCES}

[1] Paul Heymann, Georgia Koutrika,Hector Garcia-Molina on "Fighting Spam on Social Websites".

[2] Alexandros Nanopoulos on "Item Recommendation in Collaborative Tagging Systems",

Guozhu Dong, Jiawei Han, Joyce M.W. Lam,Jian Pei, KeWang,Wei Zou on "Mining Constrained Gradients in Large Databases"

[4] Jennifer Ann Golbeck,JamesHendler on "Computing And Applying Trust In Web-Based Social Networks".

[5] Jure Leskovec,DanielHuttenlocher,Jon Kleinberg on "Predicting Positive and Negative Links in Online Social Networks".

[6] MagdaliniEirinaki, Malamati D. Louta on "A Trust-Aware System for Personalized User Recommendations in Social Networks". 